\title{
Erratum: Approximate periodically focused solutions to the nonlinear Vlasov-Maxwell equations for intense beam propagation through an alternating-gradient field configuration \\ [Phys. Rev. ST Accel. Beams 2, 074401 (1999)]
}

\author{
Ronald C. Davidson, Hong Qin, and Paul J. Channell \\ (Published 7 February 2000)
}

PACS numbers: 29.27.Bd, 41.75.-i, 41.85.-p, 99.10.+g

We have detected an algebraic error in our recent paper on Hamiltonian averaging techniques in an alternatinggradient field configuration. As summarized below, the main changes are in the definitions of the coupling coefficients $\delta_{x}(s), \delta_{y}(s), \kappa_{f x}$, and $\kappa_{f y}$. With the corrected definitions, the main results of the original paper remain unchanged, including the coordinate transformation to order $\epsilon^{3}$, the transformed Hamiltonian and nonlinear Vlasov-Maxwell equations, and the back-transformed properties in the laboratory frame.

The main error occurs in the definition of the third-order Hamiltonian $\mathcal{H}_{3}$ in Eq. (50), which should include on the right-hand side of Eq. (50) the additional terms

$$
x_{2} \frac{\partial^{2} S_{1}}{\partial X \partial s}+y_{2} \frac{\partial^{2} S_{1}}{\partial Y \partial s}=-\tilde{\kappa}_{x}(s) x_{2} X-\tilde{\kappa}_{y}(s) y_{2} Y,
$$

where use has been made of Eq. (37), and $\partial S_{1} / \partial s=-(1 / 2)\left[\tilde{\kappa}_{x}(s) X^{2}+\tilde{\kappa}_{y}(s) Y^{2}\right]$. The expression for $\mathcal{H}_{3}$ in Eq. (51) is then modified to become

$$
\mathcal{H}_{3}=\beta_{x}(s)\left[X^{\prime 2}-\tilde{\kappa}_{x} X^{2}-X \frac{\partial \psi}{\partial X}\right]+\beta_{y}(s)\left[Y^{\prime 2}-\tilde{\kappa}_{y} Y^{2}-Y \frac{\partial \psi}{\partial Y}\right]+\frac{1}{2} \alpha_{x}^{2}(s) X^{2}+\frac{1}{2} \alpha_{y}^{2}(s) Y^{2}+\frac{\partial S_{3}}{\partial s} .
$$

The only changes in the analysis leading to the final expression for the slowly varying Hamiltonian $\mathcal{H}$ defined in Eq. (66) are in the definitions of the coupling coefficients $\delta_{x}(s), \delta_{y}(s),\left\langle\delta_{x}\right\rangle,\left\langle\delta_{y}\right\rangle, \kappa_{f x}$, and $\kappa_{f y}$ in Eqs. (35), (59), and (63). The corrected definitions are given by

$$
\begin{aligned}
\delta_{x}(s)=\alpha_{x}^{2}(s), & \delta_{y}(s)=\alpha_{y}^{2}(s), \\
\left\langle\delta_{x}\right\rangle=\frac{1}{S} \int_{0}^{S} d s \alpha_{x}^{2}(s), & \left\langle\delta_{y}\right\rangle=\frac{1}{S} \int_{0}^{S} d s \alpha_{y}^{2}(s), \\
\kappa_{f x}=\frac{1}{S} \int_{0}^{S} d s\left[\alpha_{x}^{2}(s)-\left\langle\alpha_{x}\right\rangle^{2}\right], & \kappa_{f y}=\frac{1}{S} \int_{0}^{S} d s\left[\alpha_{y}^{2}(s)-\left\langle\alpha_{y}\right\rangle^{2}\right],
\end{aligned}
$$

where $\alpha_{x}(s)$ and $\alpha_{y}(s)$ are defined in Eq. (35). With the corrected definitions given in Eq. (3), the functional form of the transformed Hamiltonian $\mathcal{H}\left(\tilde{X}, \tilde{Y}, \tilde{X}^{\prime}, \tilde{Y}^{\prime}, s\right)$ given correct to order $\epsilon^{3}$ in Eq. (66) remains unchanged, as does the form of the canonical transformation of coordinates in Eqs. (71)-(74), and the definitions of $\alpha_{x}(s), \alpha_{y}(s), \beta_{x}(s)$, and $\beta_{y}(s)$ in Eq. (35).

Most importantly, the detailed analysis of properties of the nonlinear Vlasov-Maxwell equations in the transformed variables and the back-transformed properties in the laboratory frame presented in Secs. IV and V remain unchanged, provided we make use of the corrected definitions of the coupling coefficients given above in Eq. (3). For example, for the case of a periodic focusing quadrupole field, $\alpha_{x}(s)=-\alpha_{y}(s)=\alpha_{q}(s) \equiv \int_{0}^{s} d s \kappa_{q}(s)$, and Eq. (3) reduces to

$$
\begin{gathered}
\delta_{x}(s)=\delta_{y}(s)=\delta_{q}(s) \equiv \alpha_{q}^{2}(s), \quad\left\langle\delta_{x}\right\rangle=\left\langle\delta_{y}\right\rangle=\left\langle\delta_{q}\right\rangle=\frac{1}{S} \int_{0}^{S} d s \alpha_{q}^{2}(s), \\
\kappa_{f x}=\kappa_{f y}=\kappa_{f q}=\frac{1}{S} \int_{0}^{S} d s\left[\alpha_{q}^{2}(s)-\left\langle\alpha_{q}\right\rangle^{2}\right],
\end{gathered}
$$

which are the definitions to be used in Eqs. (67), (68), (79), and (80) and the related analysis of the Vlasov-Maxwell equations in the case of a quadrupole focusing field.

Finally, for the example of a sinusoidal quadrupole lattice $\kappa_{q}(s)=\hat{\kappa}_{q} \sin (2 \pi s / S)$ considered in Table I, the entries related to the definition of $\delta_{q}(s)$ should be corrected to read [see Eq. (3)] 


$$
\begin{gathered}
\delta_{q}(s)=\frac{\lambda_{q}^{2}}{S^{2}}\left[1+\cos ^{2}\left(k_{s} s\right)-2 \cos \left(k_{s} s\right)\right], \quad\left\langle\delta_{q}\right\rangle=\frac{3}{2} \frac{\lambda_{q}^{2}}{S^{2}}, \\
\int_{0}^{s} d s\left[\delta_{q}(s)-\left\langle\delta_{q}\right\rangle\right]=\frac{\lambda_{q}^{2}}{2 \pi S}\left[\frac{1}{4} \sin \left(2 k_{s} s\right)-2 \sin \left(k_{s} s\right)\right], \quad \kappa_{f q} S^{2}=\frac{1}{2} \lambda_{q}^{2},
\end{gathered}
$$

where $k_{s}=2 \pi / S$ and $\lambda_{q}=\hat{\kappa}_{q} S^{2} / 2 \pi$.

We apologize for the algebraic error, but reiterate that all of the principal results of the original paper remain unchanged, including the coordinate transformation to order $\epsilon^{3}$, the transformed Hamiltonian and nonlinear VlasovMaxwell equations, and the back-transformed properties in the laboratory frame, provided we make use of the corrected coupling coefficients defined above in Eq. (3) [or Eq. (4) for the case of a periodic quadrupole field]. 